\title{
Trends in robotic surgery in Korean gynecology
}

\author{
Seungmee Lee ${ }^{1}$, Mee-Ran Kim², Seok Ju Seong ${ }^{3}$, Jiheum Paek ${ }^{4}$, Yoon Soon Lee ${ }^{5}$, Eun Ji Nam6, Yong-Man Kim \\ Young-Han Park ${ }^{8}$, Tae-Joong Kim ${ }^{9}$, Yong Beom Kim ${ }^{10}$, Tae Jin Kim ${ }^{11}$, San-hui Lee ${ }^{12}$, Chul Jung Kim ${ }^{13}$, Kyung-Do Ki ${ }^{14}$, \\ So-Jin Shin ${ }^{1}$, Chi-Heum Cho ${ }^{1}$ \\ ${ }^{1}$ Department of Obstetrics and Gynecology, Keimyung University School of Medicine, Daegu; ${ }^{2}$ Department of Obstetrics and Gynecology, Seoul St. \\ Mary's Hospital, The Catholic University of Korea, Seoul; ${ }^{3}$ Department of Obstetrics and Gynecology, CHA Gangnam Medical Center, CHA Univer- \\ sity, Seoul; ${ }^{4}$ Department of Obstetrics and Gynecology, Ajou University School of Medicine, Suwon; ${ }^{5}$ Department of Obstetrics and Gynecology, \\ Kyungpook National University Chilgok Hospital, Kyungpook National University School of Medicine, Daegu; ' ${ }^{\circ}$ Department of Obstetrics and \\ Gynecology, Yonsei University College of Medicine, Seoul; ' Department of Obstetrics and Gynecology, University of Ulsan College of Medicine, Asan \\ Medical Center, Seoul; ${ }^{8}$ Department of Obstetrics and Gynecology, Hallym University Sacred Heart Hospital, Anyang: ${ }^{9}$ Department of Obstetrics \\ and Gynecology, Samsung Medical Center, Sungkyunkwan University School of Medicine, Seoul; ${ }^{10}$ Department of Obstetrics and Gynecology, Seoul \\ National University Bundang Hospital, Seoul National University College of Medicine, Seongnam; ${ }^{11}$ Department of Obstetrics and Gynecology, \\ Konkuk University School of Medicine, Seoul; ${ }^{12}$ Department of Obstetrics and Gynecology, Yonsei University Wonju College of Medicine, Wonju; \\ ${ }^{13}$ Department of Obstetrics and Gynecology, Konyang University Hospital, Daejeon; ${ }^{14}$ Department of Obstetrics and Gynecology, Kyung Hee \\ University Hospital at Gangdong, Kyung Hee University College of Medicine, Seoul, Korea
}

Objective: The objective of this study is to discover the trends of robotic surgery in gynecology since the introduction of robotic surgery platforms in Korea.

Methods: Data for the use of robotic surgery in gynecologic diseases were collected from the 14 hospitals that perform most of the robotic gynecologic surgeries in Korea. Records of robotic surgeries from between 2006 and 2019 were selected ( $n=12,405$ ), and data including diagnoses, approach methods, and names of surgical procedures were obtained. Statistical analysis was performed, including a trend test.

Results: Overall, robotic surgery in gynecology showed an increasing trend (Spearman correlation coefficient $r=0.99$, $P<0.001$ ). The common cases in which robotic surgery was used for benign diseases were uterine leiomyoma, adnexal tumors, and adenomyosis, which accounted for $68 \%, 17 \%$, and $9 \%$ of benign cases, respectively. Common malignant diseases were cervical cancer, endometrial cancer, and ovarian cancer, accounting for $55 \%, 35 \%$, and $5 \%$ of malignant cases, respectively. This increasing trend was significantly evident in uterine leiomyoma, both single-site and multiport approaches.

Conclusion: Since robotic surgical platforms were introduced in Korea, the number of gynecologic robotic surgeries has grown rapidly. Considering these data, the use of robotic surgery is expected to grow continuously for both benign and malignant gynecological diseases. A large amount of minimal invasive surgeries are expected to be replaced with robotic surgeries.

Key Words: Robotic surgical procedures; Gynecology; Korea

- Received:September 1,2020 • Revised:September 21, 2020 •Accepted: September 21, 2020

- Correspondening author: Chi-Heum Cho

Department of Obstetrics and Gynecology, Keimyung University School of Medicine, 1035 Dalgubeol-daero, Dalseo-gu, Daegu 42601, Korea

E-mail:c0035@dsmc.or.kr

Correspondening author: So-Jin Shin

Department of Obstetrics and Gynecology, Keimyung University School of Medicine, 1035 Dalgubeol-daero, Dalseo-gu, Daegu 42601, Korea

E-mail:sjshinhope2014@gmail.com

This is an Open Access article distributed under the terms of the Creative Commons Attribution Non-Commercial License (http://creativecommons.org/ licenses/by-nc/4.0) which permits unrestricted non-commercial use, distribution, and reproduction in any medium, provided the original work is properly cited. 


\section{INTRODUCTION}

Since the introduction of the robotic surgical platform in the field of minimally invasive surgeries (MIS), robotic surgery has become a global trend. By the year 2018, 4,986 da Vinci ${ }^{\circledR}$ surgical systems (Intuitive Surgical, Sunnyvale, CA, USA) have been installed worldwide [1]. In Korea, 96 da Vinci ${ }^{\circledR}$ surgical systems have been installed since the introduction of the first robotic surgical platform in 2006, and in 2019, these systems were available in 61 hospitals.

Robotic surgery is making up an increasing portion of MIS. Robotic surgery has the same advantages as MIS, including less postoperative pain, shorter hospital stays, and shorter recovery times. It also has the added advantages of view magnification using 3D imaging, tremor filters, and other specialized instruments [2]. Based on these advantages, the scope of robotic surgery is expected to expand. Robotic surgery has led to an implementation of the spread of MIS, particularly in frail patients [3] and for highly complex interventions [4,5]. In practice, laparoscopic surgery and robotic surgery are both performed by selecting a surgeon. However, as robotic surgery techniques develop, patients' demand for it increases. In light of this, it is necessary to conduct statistical analyses on robotic surgeries performed in Korean gynecology.

It has been more than a decade since the first robotic surgical platform was adopted in Korean gynecology. Our outcome of interest was to analyze the annual change of robot utilization in both benign and malignant diseases, to compare them to each other, and to analyze the trend in the utilization of robotic systems, including the number of ports used to perform surgery in a given disease.

We analyzed trends in the field of gynecologic robotic surgery in terms of operation volumes, diagnoses, and approach methods. This analysis will help us understand and predict future changes in Korean gynecologic robotic surgery.

\section{MATERIALS AND METHODS}

\section{Data source}

By the year 2019, a total of 80 da Vinci $^{\circledR}$ surgical systems (regardless of version) were installed and used by gynecol- ogy surgeons in 63 tertiary medical centers throughout Korea. Of these, 14 medical centers voluntarily participated to provide data of patients who underwent gynecologic robotic surgery between the years 2006 and 2019 ( $n=12,405)$. The collected data was analyzed retrospectively.

Cases that were included involved the diagnosis of female genital organs using da Vinci ${ }^{\circledR} \mathrm{S}, \mathrm{Si}, \mathrm{X}, \mathrm{Xi}$, or SP by gynecologic oncology or general gynecologic specialists in the department of obstetrics and gynecology within each medical center. Patients' ages were recorded at the time of surgery. Robotic surgeries using a single-site approach includes procedures in which all of the robotic arms and ports for the assistant instrument were utilized via single incision. Robotic surgeries with multiple ports included those with additional robotic arm utilization or that used a single-site approach with an additional port for a surgical instrument manipulated by an assistant. The latter were considered multiport robotic surgeries.

Diagnoses were first categorized as benign or malignant diseases and then subcategorized according to the diagnosis code of the Health Insurance Review and Assessment Service. Benign diseases include uterine leiomyoma, adnexal tumor, adenomyosis, pelvic organ prolapse, cervical intraepithelial neoplasia, endometrial hyperplasia, endometrial polyp, cervical mass, and vaginal intraepithelial neoplasia. Malignant diseases include cervical cancer, endometrial cancer, gestational trophoblastic neoplasia, ovarian cancer, peritoneal cancer, tubal cancer, uterine cancer, vaginal cancer, and vulvar cancer. Tumors that were diagnosed as ovarian borderline tumors or stromal tumors of unknown malignant potential were categorized as malignant diseases.

\section{Statistical analysis}

SPSS version 26.0 for Windows (SPSS Inc., Chicago, IL, USA) was used to analyze the collected data. The Spearman correlation test was conducted to analyze the relationship between categorical variables. Spearman correlation analysis was conducted with $\rho$ indicating the crude Spearman coefficient. A P-value of less than 0.05 was considered statistically significant.

Gyne Robot Surg 2020;1(2):50-56 


\section{RESULTS}

The volume of overall robotic surgeries in benign gynecology and gynecologic oncology showed an increasing trend between 2006 and 2019 (Spearman correlation coefficient $r=0.99, P<0.001$ ) (Fig. 1A). Robotic surgery began in 2006 using a multiport approach. Data on single-site surgeries have been collected since 2009, because single-site surgery began after the introduction of the Si system. In 2006, 18 cases of robotic surgery were performed. In 2019, 13 years later, the volume increased to 3,860. The rate of yearly increase has been faster in the last five years, increasing by $58.6 \%$ in $2015,54.3 \%$ in $2016,36.9 \%$ in 2017, $57.6 \%$ in 2018, and 40\% in 2019 compared to each previous year in this study. The volume of overall robotic surgeries showed the biggest on-year growth in 2015 in the last 5 years.

(A)
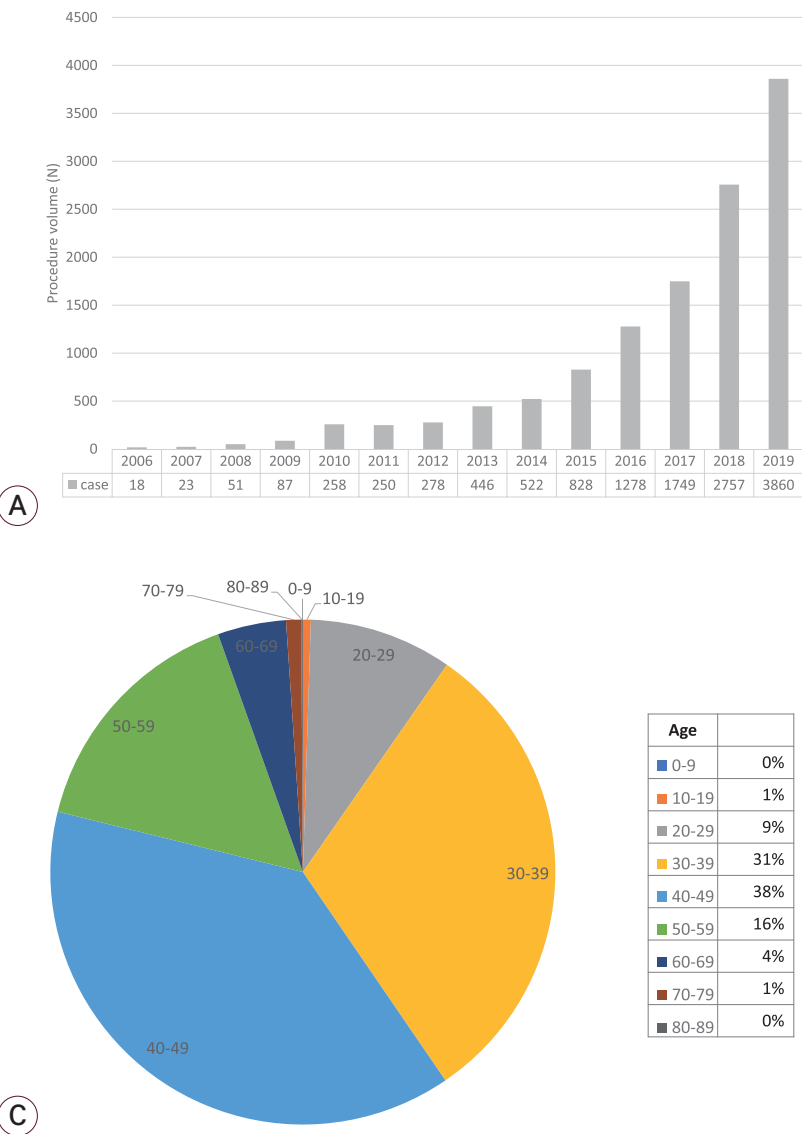

Fig. 1. Trends of robotic surgery in gynecology and gynecologic oncology by year. (A) Overall trend of robotic surgery. (B) Trend by approach, multiport versus single-site. (C) Distribution in age. (D) Trend of robotic surgery in benign and malignant diseases.
Single-site surgeries began to increase in 2014 after new da Vinci Si system was introduced and have been on the rise every year since (Fig. 1B). In 2019, 987 cases of singlesite robotic surgery were performed in Korea. The volume of single-site surgeries increased by $118.4 \%$ in $2015,94.7 \%$ in 2016, 77.6\% in 2017, 31.5\% in 2018, and 14.2\% in 2019 compared to each previous year. Multiport surgeries were performed in 18 cases in 2006, the first year that robotic surgery was introduced in Korea, and they increased by $46.7 \%$ in 2015 , $42.3 \%$ in 2016, $20.3 \%$ in $2017,73.4 \%$ in 2018, and 51.8\% in 2019 compared to each previous year. Both single-site and multiport surgeries show a growing trend in statistical analysis ( $r=0.90$ and $r=0.99$, respectively, $P<0.001)$. Patients who underwent robotic surgery were primarily in aged 30-60. There was no difference in age distribution between all gynecological surgeries (Fig. 1C).

The analysis of robotic surgery was performed according

(B)
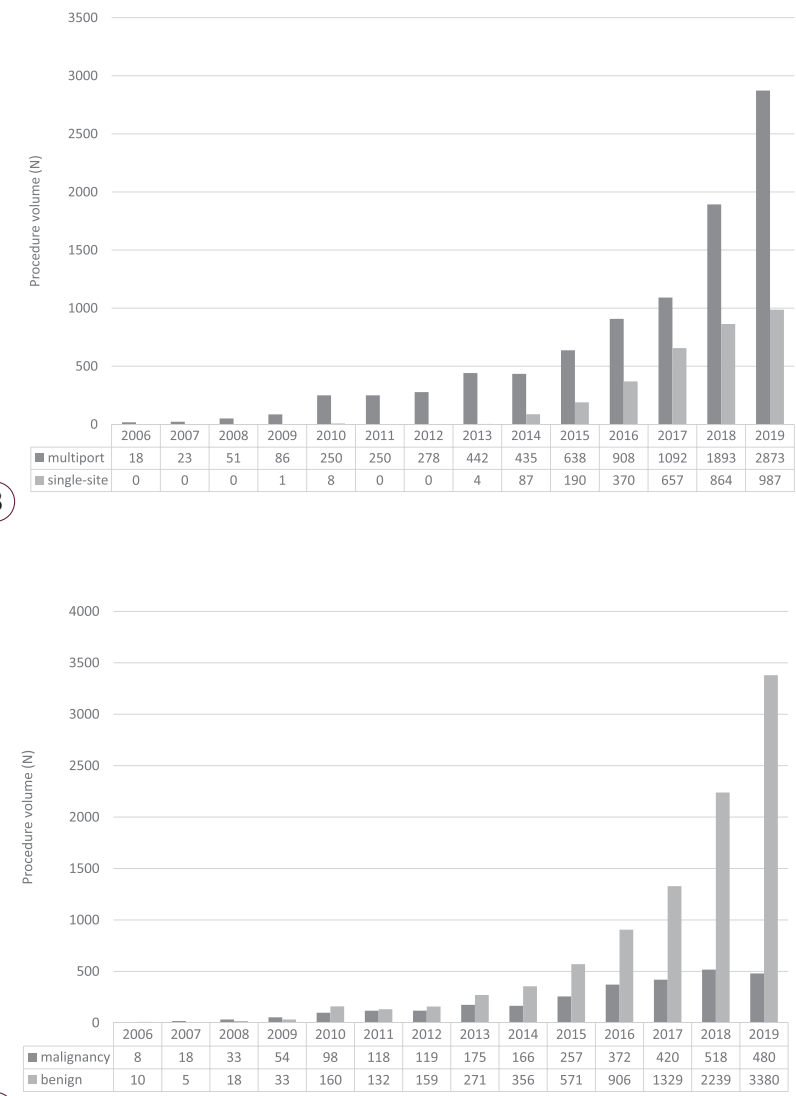

(D) 
to the type of disease. Benign diseases were a primary indication for gynecologic robotic surgery and showed an increasing trend over the study period ( $r=0.98, P<0.001$ ) (Fig. 1D). In 2019, 3,380 cases of robotic surgery were performed for benign diseases. The volume of robotic surgery for benign disease has increased significantly since 2015. The proportion of benign surgeries increased by $76.0 \%$ in $2017,81.2 \%$ in 2018, and 87.6\% in 2019 compared to each previous year. The rates of increase were $58.7 \%$ in 2016, 46.7\% in 2017, 68.5\% in 2018, and 51.0\% in 2019 compared to each previous year. For malignant diseases, the volume of robotic surgeries showed a slow but increasing trend $(r=0.99, P<0.001)$.

Uterine leiomyoma was the most common indication for gynecologic robotic surgery in benign diseases, accounting for $68 \%$ of all benign cases (Fig. 2A). Adnexal tumors and adenomyosis followed, accounting for $17 \%$ and $9 \%$ of benign cases, respectively. In malignant diseases, robotic surgery was conducted mainly in cervical cancer and endometrial cancer, accounting for $92 \%$ of all cases (47\% and $45 \%$, respectively) (Fig. 2B). Ovarian cancer accounted for $5 \%$ of malignant cases.

In the view of the platform system, both single-site and multiport surgeries increased for benign diseases (Fig. 3A).
(A)

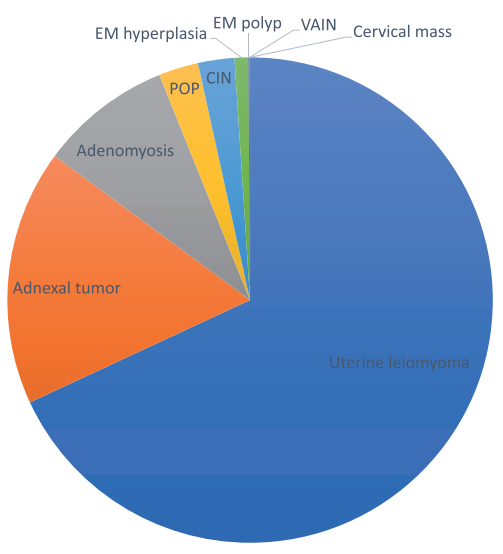

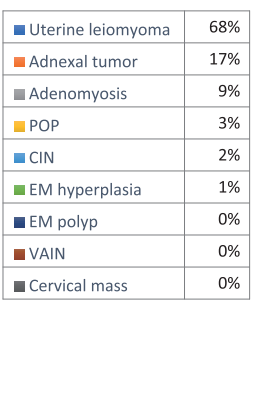

(B)

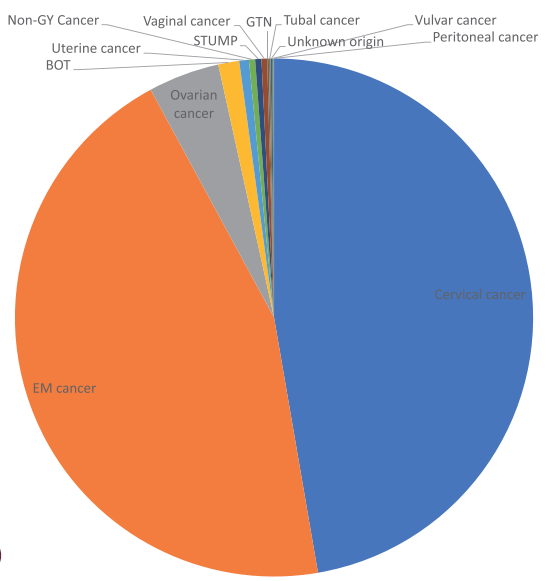

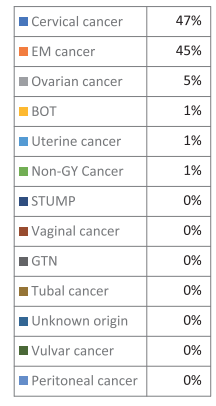

Fig. 2. Distribution of indication for gynecologic robotic surgeries. (A) Distribution in benign diseases. (B) Distribution in malignant diseases. POP, pelvic organ prolapse; CIN, cervical intraepithelial neoplasia; EM, endometrial; VAIN, vaginal intraepithelial neoplasia; BOT, borderline ovarian tumor; GY, gynecologic; STUMP, smooth muscle tumor of uncertain malignant potential; GTN, gestational trophoblastic neoplasia.

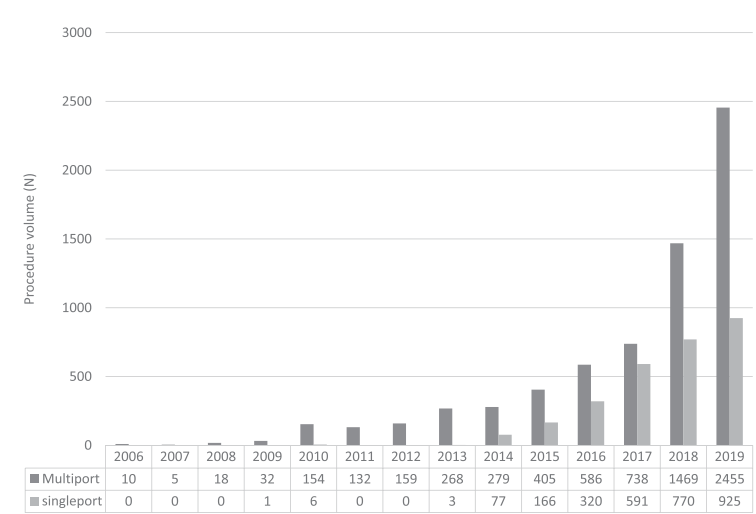

(A)

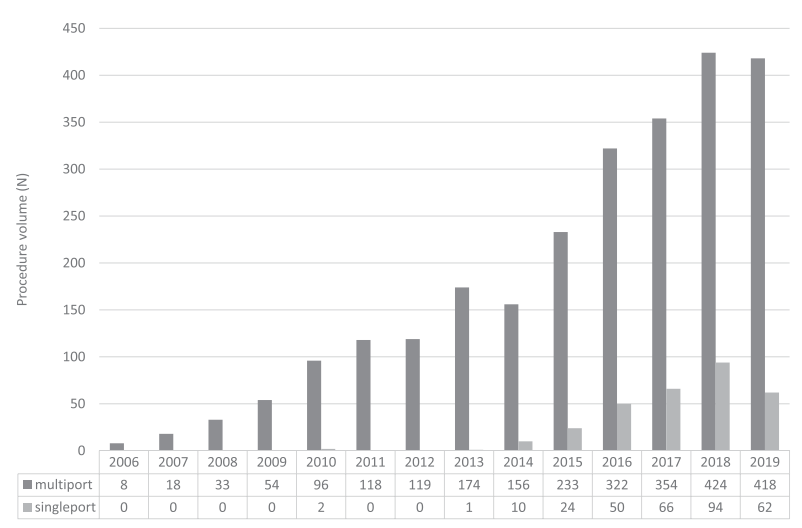

(B)

Fig. 3. Annual change of robotic surgeries in gynecology and gynecologic oncology by approach. (A) Annual change in benign diseases. (B) Annual change in malignant diseases. 


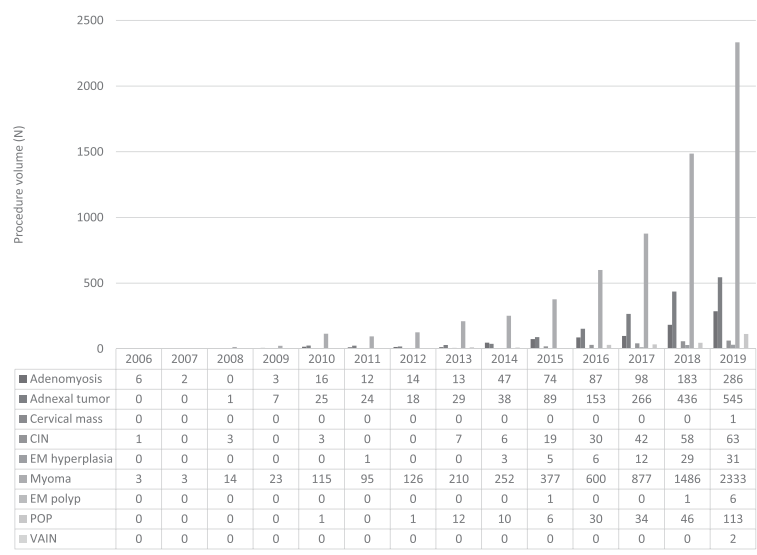

Fig. 4. Annual change of robotic surgeries in gynecology and gynecologic oncology by diagnosis. (A) Annual change in benign diseases. (B) Annual change in malignant diseases. CIN, cervical intraepithelial neoplasia; EM, endometrial; POP, pelvic organ prolapse; VAIN, vaginal intraepithelial neoplasia; BOT, borderline ovarian tumor; GY, gynecologic; STUMP, smooth muscle tumor of uncertain malignant potential; GTN, gestational trophoblastic neoplasia.

Robotic surgery for main indications increased steadily in both benign and malignant diseases. In malignant diseases, the number of robotic surgeries on both platforms continued to increase until 2018 but decreased in 2019, especially for cervical cancer. In particular, single-site surgeries showed a greater decrease (Fig. 3B). Overall, robotic surgeries showed a trend in which uterine leiomyoma and endometrial cancer were the main indications for benign and malignant diseases, respectively (Fig. 4).

\section{DISCUSSION}

In the USA, Food and Drug Administration clinical clearances were obtained for gynecologic surgical procedures in April 2005. Gynecology was the second-largest surgical specialty that used robotic surgery in the USA in 2018, with a procedure volume of approximately 265,000 cases in 2018, compared with 252,000 in 2017 and 246,000 in 2016 [1]. Also in 2018, gynecologic robotic surgery in the USA only grew 5.2\% compared to the previous year, while outside the USA, the field of robotic surgery grew rapidly, driven by urologic surgery, general surgery, and gynecologic oncologic procedures. Over the past 5 years, robotic surgeries have increased by $20 \%$ every year worldwide $[1,6]$. In Korea, the number of gynecologic procedures increased by $57.6 \%$ in 2018 and $40 \%$ in 2019 compared to each previous year in this study. This trend is much faster than the global trend. Needs of minimal scar on surgeries has increased, causing the volume of MIS to grow steadily. The demand for robotic platforms, for which young people are quickly adapting, is rapidly increasing for benign diseases. In particular, robotic surgery for uterine leiomyoma has shown a rapid rise. In myomectomy, the advantage of robotic surgery over laparoscopy is that sutures are more accessible and easier to experience. In Korea, the number of patients treated for uterine myoma in women in reproductive age (aged 20-50) is increasing every year. Ministry of Health and Welfare reported that the number of uterine myoma patients increased 21\% in 2009 compared by 2004 [7] and number of patients and medical cost was steadily increased between 2009 and 2013 [8]. As the age of pregnancy has increased, the number of uterine leiomyoma patients who need surgery to preserve their fertility has also increased, in turn affecting the frequency of robotic surgery for uterine leiomyoma.

In the past 5 years, robotic surgery showed significant growth in gynecologic oncology. Endometrial and cervical cancer with MIS contributed to this growth. However, the reduction of robotic surgery for cervical cancer in 2019 is noticeable (Fig. 4B). As the result of the "Laparoscopic Approach to Carcinoma of the Cervix (LACC) trial," concerns about oncological outcomes led to the need for more data collection and analysis, resulting in a decrease in robotic surgery for cervical cancer [9]. The results of the LACC trial 
caused controversy in that the oncological outcome of MIS was lower than that of laparotomic surgery. The controversy is that the recurrence rate was lower than that in other studies, and the follow-up period of the study was short, so the proficiency of the surgeons performing the MIS has been questioned [10-12]. For this reason, there has been an effort to identify and select patients who may have lower risks with MIS. In Europe, a robotic surgery group is currently conducting a "Robotic-assisted Approach to Cervical Cancer (RACC) trial" to compare the oncologic outcomes of open surgery and robotic surgery in early cervical cancer [13]. To clarify the role of MIS in treating cervical cancer, it is necessary to wait for the results of other studies that are currently ongoing.

Despite all of this, the utility of robotic surgery for endometrial cancer is notable, as many studies have reported the efficacy of robotic surgery in endometrial cancer already. A randomized controlled trial conducted in Finland compared the surgical outcomes of laparoscopy with robotic surgery in endometrial cancer [14]. The trial's result showed that the outcomes were similar between the groups, but robotic surgery improved patients' quality of life, with benefits noted up to 12 weeks after surgery [15]. Bernardini et al. reported that robotic surgery would be a safe and effective option in the surgical treatment of endometrial cancer, especially in obese women [16-18].

Robotic surgery is more beneficial for less experienced surgeons because it allows faster and more accurate training compared to laparoscopic surgery [19,20]. The benefits of robotic surgery are further reinforced by the application of improved instruments and docking methods whenever a new robotic system is developed. These contributed to the rapid installation of robotic systems in hospitals. In Korea, the major obstacle to the expansion of robotic surgery may be a concern about price [21]. Patients with private insurance are less burdened with medical costs, and robotic surgery is more accessible to them. Reducing the cost of robotic surgery will improve accessibility for all patients.

Given the advantages of MIS and robotic surgery, Korean surgeons and patients have a high preference for it. As fast as the volume of robotic surgery is growing, the MIS platform is replacing conventional laparoscopy with robotic surgery. Nevertheless, conventional laparoscopy has its own advantages, so the choice of robotic or laparoscopic surgery should be based on proper indications before an operation is conducted.

When robotic surgery was first introduced, laparoscopic surgery had already become a potent platform for MIS, and some devalued the utility of robots. Now, however, robotic surgery is thought to have become alternative form of MIS equivalent to laparoscopic surgery.

\section{Conflict of interest}

No potential conflict of interest relevant to this article was reported.

\section{References}

1. Intuitive Surgical. Annual Report 2018 [Internet]. Sunnyvale (CA): Intuitive Surgical; c2019 [cited 2020 Jul 28]. Available from: http:// www.annualreports.com/HostedData/AnnualReportArchive/i/ NASDAQ_ISRG_2018.pdf.

2. Bouquet de Joliniere J, Librino A, Dubuisson JB, Khomsi F, Ben Ali N, Fadhlaoui A, et al. Robotic surgery in gynecology. Front Surg 2016;3:26.

3. Gallotta V, Conte C, D'Indinosante M, Federico A, Biscione A, Vizzielli G, et al. Robotic surgery in elderly and very elderly gynecologic cancer patients. J Minim Invasive Gynecol 2018;25:872-7.

4. Gallotta V, Chiantera V, Conte C, Vizzielli G, Fagotti A, Nero C, et al. Robotic radical hysterectomy after concomitant chemoradiation in locally advanced cervical cancer: a prospective phase II study. J Minim Invasive Gynecol 2017;24:133-9.

5. Gallotta V, Cicero C, Conte C, Vizzielli G, Petrillo M, Fagotti A, et al. Robotic versus laparoscopic staging for early ovarian cancer: a case-matched control study. J Minim Invasive Gynecol 2017;24:293-8.

6. Intuitive Surgical. Annual Report 2015 [Internet]. Sunnyvale (CA): Intuitive Surgical; c2016 [cited 2020 Jul 28]. Available from: http:// www.annualreports.com/HostedData/AnnualReportArchive/i/ NASDAQ_ISRG_2015.pdf.

7. Ministry of Health and Welfare. Press release [Internet]. Sejong (KR): Ministry of Health and Welfare; c2010 [cited 2020 Sep 19]. Available from: http://www.mohw.go.kr/react/al/sal0301vw.jsp?PAR_ MENU_ID=04\&MENU_ID=0403\&CONT_SEQ=241055.

8. Ministry of Health and Welfare. Press release [Internet]. Sejong (KR): Ministry of Health and Welfare; c2015 [cited 2020 Sep 19]. Available from: http://www.mohw.go.kr/react/al/sal0301vw.jsp?PAR_ MENU_ID=04\&MENU_ID=0403\&CONT_SEQ=316446.

9. Ramirez PT, Frumovitz M, Pareja R, Lopez A, Vieira M, Ribeiro R, et al. Minimally invasive versus abdominal radical hysterectomy for cervical cancer. N Engl J Med 2018;379:1895-904.

10. Kanao H, Aoki Y, Takeshima N. Unexpected result of minimally in- 
vasive surgery for cervical cancer. J Gynecol Oncol 2018;29:e73.

11. Kimmig R, Ind T. Minimally invasive surgery for cervical cancer: consequences for treatment after LACC study. J Gynecol Oncol 2018;29:e75

12. Park JY, Nam JH. How should gynecologic oncologists react to the unexpected results of LACC trial? J Gynecol Oncol 2018;29:e74.

13. National Library of Medicine. Robot-assisted Approach to Cervical Cancer (RACC) [Internet]. Bethesda (MD): National Library of Medicine: c2019 [cited 2020 Jul 3]. Available from: https://clinicaltrials.gov/ct2/show/NCT03719547.

14. Mäenpää MM, Nieminen K, Tomás El, Laurila M, Luukkaala TH, Mäenpää JU. Robotic-assisted vs traditional laparoscopic surgery for endometrial cancer: a randomized controlled trial. Am J Obstet Gynecol 2016;215:588.e1-588.e7.

15. Ferguson SE, Panzarella T, Lau S, Gien LT, Samouëlian V, Giede C, et al. Prospective cohort study comparing quality of life and sexual health outcomes between women undergoing robotic, laparoscopic and open surgery for endometrial cancer. Gynecol Oncol 2018;149:476-83.

16. Bernardini MQ, Gien LT, Tipping H, Murphy J, Rosen BP. Surgical outcome of robotic surgery in morbidly obese patient with endometrial cancer compared to laparotomy. Int J Gynecol Cancer 2012;22:76-81.
17. Cusimano MC, Simpson AN, Dossa F, Liani V, Kaur Y, Acuna SA, et al. Laparoscopic and robotic hysterectomy in endometrial cancer patients with obesity: a systematic review and metaanalysis of conversions and complications. Am J Obstet Gynecol 2019;221:410-28.e19.

18. Stephan JM, Goodheart MJ, McDonald M, Hansen J, Reyes HD, Button A, et al. Robotic surgery in supermorbidly obese patients with endometrial cancer. Am J Obstet Gynecol 2015;213:49.e149.e8.

19. Heemskerk J, van Gemert WG, de Vries J, Greve J, Bouvy ND. Learning curves of robot-assisted laparoscopic surgery compared with conventional laparoscopic surgery: an experimental study evaluating skill acquisition of robot-assisted laparoscopic tasks compared with conventional laparoscopic tasks in inexperienced users. Surg Laparosc Endosc Percutan Tech 2007;17:171-4.

20. Chandra V, Nehra D, Parent R, Woo R, Reyes R, HernandezBoussard T, et al. A comparison of laparoscopic and robotic assisted suturing performance by experts and novices. Surgery 2010;147:830-9.

21. Wright JD, Ananth CV, Lewin SN, Burke WM, Lu YS, Neugut AI, et al. Robotically assisted vs laparoscopic hysterectomy among women with benign gynecologic disease. JAMA 2013;309:68998. 